\title{
The first record of Cucullia umbratica (Lepidoptera: Noctuidae) from Japan
}

\author{
Soichiro Noguchi ${ }^{\ddagger}$, Takahiro Ochiai§ \\ ‡ Systematic Entomology, Graduate School of Agriculture, Hokkaido University, Sapporo, Japan \\ $\S$ Wood Chemistry and Chemical Biology, Graduate School of Agriculture, Hokkaido University, Sapporo, Japan
}

\author{
Corresponding author: Soichiro Noguchi (soichiro0127@eis.hokudai.ac.jp), \\ Takahiro Ochiai (takahiro.ochiai0106@gmail.com) \\ Academic editor: Shinichi Nakahara \\ Received: 28 Feb 2019 | Accepted: 19 Mar 2019 | Published: 10 Apr 2019 \\ Citation: Noguchi S, Ochiai T (2019) The first record of Cucullia umbratica(Lepidoptera: Noctuidae) from Japan. \\ Biodiversity Data Journal 7: e34197. https://doi.org/10.3897/BDJ.7.e34197
}

\begin{abstract}
\section{Background}

Cucullia umbratica is known from Europe (from Spain to southern Fennoscandia), Russia, Afghanistan, Turkestan and Mongolia, in the Palearctic. In addition, introduction of this species to Canada has been reported recently.
\end{abstract}

\section{New information}

We report this species from Japan for the first time, from two locations at Hokkaidō. The earliest record is from 2015.

\section{Keywords}

COI, Cucullia, Genbank, Hokkaidō, Japan, Lepidoptera, Noctuidae 


\section{Introduction}

Cucullia umbratica (Linnaeus, 1758) is a fairly large species of moth, with a wingspan of 48-57 mm (Heath and Emmet 1983). Due to its peculiar appearance, this species is generally called the "shark" moth (Price 2017). The larvae of this species utilise a wide range of Asteraceae as host plants (Hampson 1906, Heath and Emmet 1983). Although this species is widely distributed throughout the Palearctic (Hampson 1906, Heath and Emmet 1983, Dubatolov and Matov 2009), it has not been recorded from Japan to date (Kishida 2011, Jinbo 2017). In the present paper, we report this species from Hokkaidō for the first time and this also represents the first record of this species from Japan.

\section{Materials and methods}

\section{Specimens}

Dried specimens were used. The genitalia were dissected and stained with safranin and Fast Blue. Genital structures were observed under a Nikon SMZ 745T microscope. Digital images of the specimens were captured using a Nikon D5600 digital camera body and a Tamron SP $90 \mathrm{~mm}$ F2.8 Di MACRO lens and genitalia images were captured using a Canon EOS 5D Mark IV camera body with a Canon EF 100 mm F2.8 L IS USM Macro lens. The field photo was taken with a Canon EOS 6D camera body with a Canon EF 100mm F2.8 Macro lens and a Canon Macro Ring Lite ML-3. The morphological terminology used herein generally follows that of Heath (1976).

\section{Molecular methods}

DNA was extracted from the distal portion of the 8th abdominal segment and the entire sample was suspended in extraction buffer following manufacturer's protocols for the DNeasy Blood \& Tissue Kit (Qiagen, Inc., Valencia, CA, U.S.A.). The specimen used for DNA extraction was deposited in the insect collection at the Hokkaido University Museum (SEHU), Sapporo, Japan. Polymerase chain reaction (PCR) amplification of cytochrome oxidase I (COI) was carried out using an Applied Biosystems 2720 Thermal Cycler (Applied Biosystems, Foster City, CA, USA) with a touchdown amplification programme: 2 minutes at $94^{\circ} \mathrm{C}, 10 \mathrm{~s}$ at $94^{\circ} \mathrm{C}, 10 \mathrm{~s}$ at $55-46^{\circ} \mathrm{C}, 105 \mathrm{~s}$ at $68^{\circ} \mathrm{C}, 36$ cycles of $10 \mathrm{~s}$ at $94^{\circ} \mathrm{C}, 10 \mathrm{~s}$ at $45^{\circ} \mathrm{C}, 105 \mathrm{~s}$ at $68^{\circ} \mathrm{C}$ and a final extension step for 2 minutes at $72^{\circ} \mathrm{C}$. A single fragment of $1536 \mathrm{bp}$ was amplified from the PCR amplification using primer pair CO1-exF (5ATCGCCTAAACTTCAGCCATT-3) and TL-N-3017 (5-CTTAAATCCATTGCACTAATCTGCCA TA-3) (Scheffer et al. 2004). PCR products were visualised via $1 \%$ agarose gel electrophoresis with ethidium bromide to confirm amplification. PCR products were purified with innuPREP PCRpure Light Kit (analytikjena, Jena, Germany) according to the manufacturer's instructions. Purified PCR products were quantified using a Nanodrop One (Nanodrop Technologies, Wilmington, DE, USA). Purified products were sequenced in both directions using BigDye Terminator v3.1 chemistry (Applied Biosystems, Foster City, CA, USA). Primers used for sequencing were six primer sets (Table 1). The sequencing 
products were purified on Sephadex G-50 Fine DNA Grade (GE Healthcare, Chicago, U.S.A.) and analysed in 3500xL Genetic Analyzer (Applied Biosystems, Foster City, CA, USA).

\section{Table 1.}

Primers used for sequencing

\begin{tabular}{|l|l|l|}
\hline Primer name & Sequence $\mathbf{( 5}^{\prime} \mathbf{-} \mathbf{3}^{\prime} \mathbf{)}$ & Source \\
\hline CO1-exF & ATCGCCTAAACTTCAGCCATT & Present study \\
\hline CO1-GK.1R & ACTGCACCTAAAATTGATGA & Present study \\
\hline CO1-Hp.1F & AGCTGGAACAGGATGAAC & Present study \\
\hline CO1-Hp.3R & TAGCAAAAACAGCTCCTA & Present study \\
\hline CO1-Hp.3F & CTCTTCATGATACTTATTATG & Present study \\
\hline TL-N-3017 & CTTAAATCCATTGCACTAATCTGCCATA & Scheffer et al. 2004 \\
\hline
\end{tabular}

\section{Sequence Analysis}

Sequence contigs were assembled using BioLign Version 4.0.6.2 (2005; http://en.biosoft.net/dna/BioLign.html) and aligned consensus sequences were created using BioEdit 7.0.5.3 (Hall 1999). The p-distance was calculated by comparing with $13 \mathrm{COI}$ sequences registered in GenBank (accession numbers: KJ389042, GU686885, GU686845, KX043158, JF415531, GU828701, JF415532, JF415530, GU654999, KX040689, KM573523, $\mathrm{HQ563405}$ and KJ183425) using MEGA (Molecular Evolutionary Genetics Analysis) Version 7.0 (Kumar et al. 2016).

\section{Taxon treatment}

\section{Cucullia umbratica Linnaeus, 1758}

\section{Materials}

a. scientificName: Cucullia umbratica; kingdom: Animalia; phylum: Euarthropoda; class: Insecta; order: Lepidoptera; family: Noctuidae; genus: Cucullia; specificEpithet: umbratica; scientificNameAuthorship: Linnaeus; country: Japan; stateProvince: Hokkaidō; county: Sōya-gun; municipality: Sarufutsu Village; locality: Hama-sarufutsu; samplingProtocol: light trap; eventDate: 2015-07-18; individualCount: 1; sex: male; lifeStage: adult; recordedBy: Takuya Ito; identifiedBy: Takuya Ito; dateldentified: 2015

b. scientificName: Cucullia umbratica; kingdom: Animalia; phylum: Euarthropoda; class: Insecta; order: Lepidoptera; family: Noctuidae; genus: Cucullia; specificEpithet: umbratica; scientificNameAuthorship: Linnaeus; country: Japan; stateProvince: Hokkaidō; county: Sōya-gun; municipality: Sarufutsu Village; locality: Hama-sarufutsu; samplingProtocol: light trap; eventDate: 2015-08-01; fieldNotes: DNA extraction; individualCount: 1; sex: 
male; lifeStage: adult; catalogNumber: 87764; recordedBy: Takuya Ito; identifiedBy: Takuya Ito; dateldentified: 2015; institutionCode: SEHU; collectionCode: Insect

c. scientificName: Cucullia umbratica; kingdom: Animalia; phylum: Euarthropoda; class: Insecta; order: Lepidoptera; family: Noctuidae; genus: Cucullia; specificEpithet: umbratica; scientificNameAuthorship: Linnaeus; country: Japan; stateProvince: Hokkaidō; county: Sōya-gun; municipality: Sarufutsu Village; locality: Hama-sarufutsu; samplingProtocol: light trap; eventDate: 2016-07-23; fieldNotes: DNA extraction, DDBJ: LC460439; individualCount: 1; sex: male; lifeStage: adult; catalogNumber: 87761; recordedBy: Takuya Ito; identifiedBy: Takuya Ito; dateldentified: 2016; institutionCode: SEHU; collectionCode: Insect

d. scientificName: Cucullia umbratica; kingdom: Animalia; phylum: Euarthropoda; class: Insecta; order: Lepidoptera; family: Noctuidae; genus: Cucullia; specificEpithet: umbratica; scientificNameAuthorship: Linnaeus; country: Japan; stateProvince: Hokkaidō; county: Sōya-gun; municipality: Sarufutsu Village; locality: Hama-sarufutsu; samplingProtocol: light trap; eventDate: 2016-07-23; individualCount: 1; sex: male; lifeStage: adult; recordedBy: Takahiro Ochiai; identifiedBy: Takuya Ito; dateldentified: 2016

e. scientificName: Cucullia umbratica; kingdom: Animalia; phylum: Euarthropoda; class: Insecta; order: Lepidoptera; family: Noctuidae; genus: Cucullia; specificEpithet: umbratica; scientificNameAuthorship: Linnaeus; country: Japan; stateProvince: Hokkaidō; county: Sōya-gun; municipality: Sarufutsu Village; locality: Hama-sarufutsu; samplingProtocol: light trap; eventDate: 2016-07-23; individualCount: 1; sex: male; lifeStage: adult; recordedBy: Takahiro Ochiai; identifiedBy: Takuya Ito; dateldentified: 2016

f. ScientificName: Cucullia umbratica; kingdom: Animalia; phylum: Euarthropoda; class: Insecta; order: Lepidoptera; family: Noctuidae; genus: Cucullia; specificEpithet: umbratica; scientificNameAuthorship: Linnaeus; country: Japan; stateProvince: Hokkaidō; county: Sōya-gun; municipality: Sarufutsu Village; locality: Hama-sarufutsu; samplingProtocol: light trap; eventDate: 2017-07-22; individualCount: 1; sex: male; lifeStage: adult; recordedBy: Takuya Ito; identifiedBy: Takuya Ito; dateldentified: 2017

g. scientificName: Cucullia umbratica; kingdom: Animalia; phylum: Euarthropoda; class: Insecta; order: Lepidoptera; family: Noctuidae; genus: Cucullia; specificEpithet: umbratica; scientificNameAuthorship: Linnaeus; country: Japan; stateProvince: Hokkaidō; county: Sōya-gun; municipality: Sarufutsu Village; locality: Hama-sarufutsu; samplingProtocol: light trap; eventDate: 2017-07-22; individualCount: 1; sex: male; lifeStage: adult; recordedBy: Takahiro Ochiai; identifiedBy: Takuya Ito; dateldentified: 2017

h. scientificName: Cucullia umbratica; kingdom: Animalia; phylum: Euarthropoda; class: Insecta; order: Lepidoptera; family: Noctuidae; genus: Cucullia; specificEpithet: umbratica; scientificNameAuthorship: Linnaeus; country: Japan; stateProvince: Hokkaidō; county: Sōya-gun; municipality: Sarufutsu Village; locality: Hama-sarufutsu; samplingProtocol: light trap; eventDate: 2017-07-22; fieldNotes: DNA extraction; individualCount: 1; sex: male; lifeStage: adult; catalogNumber: 87763; recordedBy: Soichiro Noguchi; identifiedBy: Takuya Ito; dateldentified: 2017; institutionCode: SEHU; collectionCode: Insect

i. scientificName: Cucullia umbratica; kingdom: Animalia; phylum: Euarthropoda; class: Insecta; order: Lepidoptera; family: Noctuidae; genus: Cucullia; specificEpithet: umbratica; scientificNameAuthorship: Linnaeus; country: Japan; stateProvince: Hokkaidō; county: Esashi-gun; municipality: Hamatonbetsu Town; locality: Aza-usotan; samplingProtocol: light trap; eventDate: 2017-07-22; fieldNotes: DNA extraction; individualCount: 1; sex: female; lifeStage: adult; catalogNumber: 87762; recordedBy: Soichiro Noguchi; identifiedBy: Takuya Ito; dateldentified: 2017; institutionCode: SEHU; collectionCode: Insect 
j. $\quad$ scientificName: Cucullia umbratica; kingdom: Animalia; phylum: Euarthropoda; class: Insecta; order: Lepidoptera; family: Noctuidae; genus: Cucullia; specificEpithet: umbratica; scientificNameAuthorship: Linnaeus; country: Japan; stateProvince: Hokkaidō; county: Sōya-gun; municipality: Sarufutsu Village; locality: Hama-sarufutsu; samplingProtocol: light trap; eventDate: 2017-07-29; individualCount: 1; sex: male; lifeStage: adult; recordedBy: Takuya Ito; identifiedBy: Takuya Ito; dateldentified: 2017

k. scientificName: Cucullia umbratica; kingdom: Animalia; phylum: Euarthropoda; class: Insecta; order: Lepidoptera; family: Noctuidae; genus: Cucullia; specificEpithet: umbratica; scientificNameAuthorship: Linnaeus; country: Japan; stateProvince: Hokkaidō; county: Sōya-gun; municipality: Sarufutsu Village; locality: Hama-sarufutsu; samplingProtocol: light trap; eventDate: 2018-07-28; individualCount: 1; sex: male; lifeStage: adult; recordedBy: Takahiro Ochiai; identifiedBy: Takuya Ito; dateldentified: 2018

I. scientificName: Cucullia umbratica; kingdom: Animalia; phylum: Euarthropoda; class: Insecta; order: Lepidoptera; family: Noctuidae; genus: Cucullia; specificEpithet: umbratica; scientificNameAuthorship: Linnaeus; country: Japan; stateProvince: Hokkaidō; county: Sōya-gun; municipality: Sarufutsu Village; locality: Hama-sarufutsu; samplingProtocol: light trap; eventDate: 2018-07-28; individualCount: 1; sex: male; lifeStage: adult; recordedBy: Soichiro Noguchi; identifiedBy: Takuya Ito; dateldentified: 2018

m. scientificName: Cucullia umbratica; kingdom: Animalia; phylum: Euarthropoda; class: Insecta; order: Lepidoptera; family: Noctuidae; genus: Cucullia; specificEpithet: umbratica; scientificNameAuthorship: Linnaeus; country: Japan; stateProvince: Hokkaidō; county: Esashi-gun; municipality: Hamatonbetsu Town; locality: Aza-usotan; samplingProtocol: light trap; eventDate: 2018-07-28; individualCount: 1; sex: male; lifeStage: adult; recordedBy: Takahiro Ochiai; identifiedBy: Takuya Ito; dateldentified: 2018

\section{Diagnosis}

Cucullia umbratica can be distinguished from congeners by a combination of the following characters (Figs 1, 2): wingspan 48-57 mm; head and thorax grey mixed with brown; antenna with dorso-basally white; abdomen grey or brown; patagium elongate and forming a thoracic cowl; forewing ashen grey, the veins with dark brown; reniform and orbicular stigmata almost obsolete; basal steak to one-third of forewing, black and fine; terminal fascia dark brown but incomplete; the veins not extending into cilia; cilia white; hindwing white, the veins with brown but the latter sometimes reduced, in female the whole terminal half of hindwing brown; cilia white; terminal shade white with broken dark dividing line (Hampson 1906, Heath and Emmet 1983).

\section{Distribution}

Europe (from Spain to southern Fennoscandia), Russia, Afghanistan, Turkestan, Mongolia, North America (Hampson 1906, Heath and Emmet 1983, Dubatolov and Matov 2009, Handfield and Handfield 2010) and Japan (present study). 

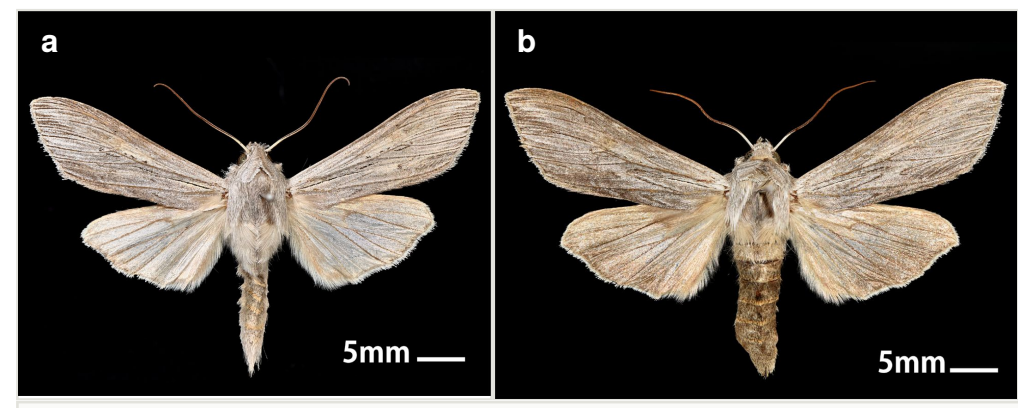

Figure 1.

Cucullia umbratica.

a: male collected at Hama-sarufutsu, Sarufutsu-village, Sōya-gun, Hokkaidō, Japan.

b: female collected at Aza-usotan, Hamatonbetsu-chō, Esashi-gun, Hokkaidō, Japan.

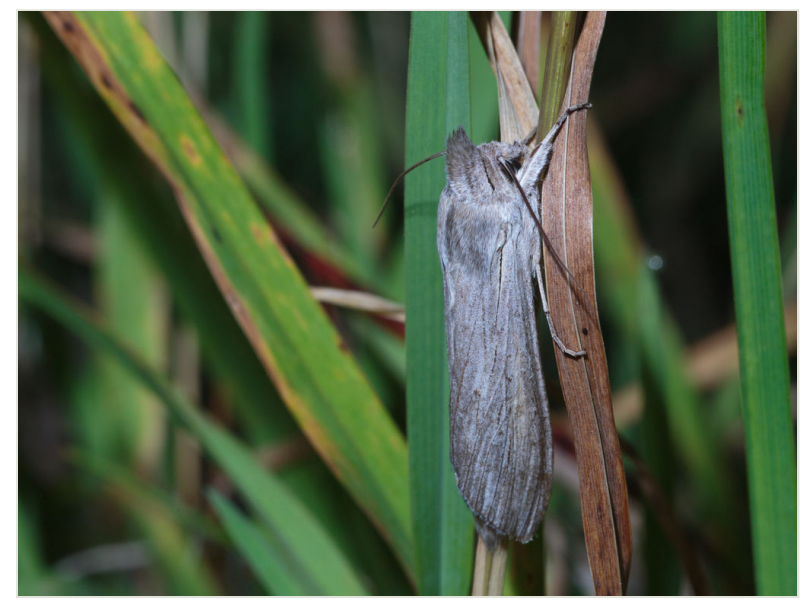

Figure 2. doi

Cucullia umbratica, in Hama-sarufutsu, Sarufutsu-village, Sōya-gun, Hokkaidō, Japan. Photo by Takahiro Ochiai.

\section{Discussion}

We have collected Cucullia umbratica every year in Sarufutsu village since 2015 and in Hamatonbetsu-chō since 2017. Therefore, this species is considered to be established at least around the Sarufutsu and Hamatonbetsu areas. We have collected 12 males and one female since 2015. These specimens have a wingspan of $47.5-53 \mathrm{~mm}$, these sizes agreeing with Heath and Emmet (1983). Other characters also agree with the species diagnosis as shown in Hampson (1906), Heath and Emmet (1983). Especially, the genital structures are in complete agreement with those shown in Heath and Emmet (1983), 
Handfield and Handfield (2010). From the combination of the general and genital morphology (Fig. 3), we identified the species we collected as Cucullia umbratica.

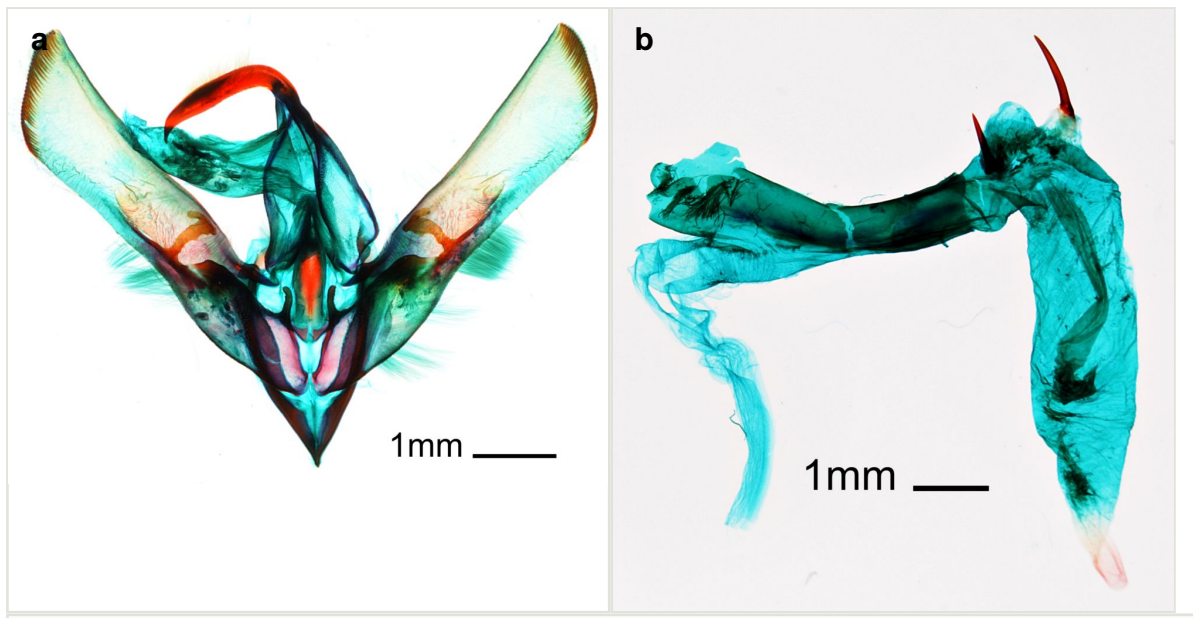

Figure 3.

Male genitaria of Cucullia umbratica from Hama-sarufutsu, Sarufutsu village, Sōya-gun, Hokkaidō, Japan.

a: male genitalia, ventral view with aedeagus removed. doi

b: aedeagus with vesica everted. doi

We also sequenced the entire $\mathrm{COI}$ gene (1536 bp) of an individual collected at Hamasarufutsu, Sarufutsu village, Sōya-gun, Hokkaidō, Japan, 23 July 2016, which was registered at DDBJ under the accession number LC460439. The preliminary comparisons showed that the sequence obtained from Hokkaidō is very close to the other registered COI sequences of Cucullia umbratica, collected from Europe (p-distances 0.000-0.005), Canada (0.002) and China (0.033) (Table 2). Although the COI sequence obtained from the Japanese sample was almost identical with that obtained from European and Canadian samples (the latter is thought to have been introduced from Europe: Handfield and Handfield 2010), p-distance between Japanese and Chinese samples was significantly high. If this genetic difference corresponds to the genetic differentiation between European and Asian populations of Cucullia umbratica, the present result provides support for the possibility that the Japanese population of this moth was introduced from Europe. However, the genetic distance, greater than $3 \%$ between European and Chinese populations, is far beyond the range of generally recognised intraspecific variations of Lepidoptera (Hebert et al. 2004) and the basis of the species identification for the Chinese specimen was not provided (Jin et al. 2018). Therefore, it might be possible that the COI barcode of Chinese Cucullia umbratica (Genbank accession number $\underline{\mathrm{KJ} 183425}$ ) has been obtained from a misidentified specimen. Further research is necessary in the future to elucidate the intraspecific genetic structure of Cucullia umbratica and the origin of the Japanese population. 
Table 2.

p-distances

\begin{tabular}{|c|c|c|c|c|c|c|c|c|c|c|c|c|c|c|}
\hline Acc. Number & 1 & 2 & 3 & 4 & 5 & 6 & 7 & 8 & 9 & 10 & 11 & 12 & 13 & 14 \\
\hline \multicolumn{14}{|l|}{$\begin{array}{l}\text { 1. Present } \\
\text { study }\end{array}$} & \\
\hline 2. KJ389042 & 0.002 & & & & & & & & & & & & & \\
\hline 3. GU686885 & 0.005 & 0.004 & & & & & & & & & & & & \\
\hline 4. GU686845 & 0.002 & 0.000 & 0.004 & & & & & & & & & & & \\
\hline 5. $\mathrm{KX043158}$ & 0.002 & 0.000 & 0.004 & 0.000 & & & & & & & & & & \\
\hline 6. JF415531 & 0.002 & 0.000 & 0.004 & 0.000 & 0.000 & & & & & & & & & \\
\hline 7. GU830689 & 0.004 & 0.002 & 0.005 & 0.002 & 0.002 & 0.002 & & & & & & & & \\
\hline 8. JF415532 & 0.002 & 0.000 & 0.004 & 0.000 & 0.000 & 0.000 & 0.002 & & & & & & & \\
\hline 9. JF415530 & 0.002 & 0.000 & 0.004 & 0.000 & 0.000 & 0.000 & 0.002 & 0.000 & & & & & & \\
\hline 10. GU654999 & 0.002 & 0.000 & 0.004 & 0.000 & 0.000 & 0.000 & 0.002 & 0.000 & 0.000 & & & & & \\
\hline 11. KX040689 & 0.002 & 0.000 & 0.004 & 0.000 & 0.000 & 0.000 & 0.002 & 0.000 & 0.000 & 0.000 & & & & \\
\hline 12. $\mathrm{KM} 573523$ & 0.002 & 0.000 & 0.004 & 0.000 & 0.000 & 0.000 & 0.002 & 0.000 & 0.000 & 0.000 & 0.000 & & & \\
\hline 13. $\underline{\mathrm{HQ} 563405}$ & 0.000 & 0.002 & 0.005 & 0.002 & 0.002 & 0.002 & 0.004 & 0.002 & 0.002 & 0.002 & 0.002 & 0.002 & & \\
\hline 14. KJ183425 & 0.033 & 0.035 & 0.035 & 0.035 & 0.035 & 0.035 & 0.033 & 0.035 & 0.035 & 0.035 & 0.035 & 0.035 & 0.033 & \\
\hline
\end{tabular}

\section{Acknowledgements}

Authors are grateful to Mr. Takuya Ito, Hokkaido Institute of Public Health, for his kind assistance in collecting Cucullia umbratica and for supplying genetic data. Thanks are also due to Dr. Kazunori Yoshizawa, Systematic Entomology, Hokkaido University, for his critical comments, to Alyssa Li Suzumura, Systematic Entomology, Hokkaido University, for editing the English and to Dr. Ryan St Laurent, University of Florida and Dr. Zhaofu Yang, Northwest A \& F University, for editing the English and their useful comments and advice concerning the manuscript.

\section{References}

- Dubatolov VV, Matov AY (2009) Noctuids (Insecta, Lepidoptera, Noctuidae) of Lower Amur. Amurian Zoological Journal 1 (4): 327-373. [In Russian].

- Hall T (1999) BioEdit: a user-friendly biological sequence alignment editor and analysis program for Windows 95 / 98 / NT. Nucleic Acids 41: 95-98. 
- Hampson G (1906) Catalogue of the Lepidoptera Phalaenae in the Collection of the British Museum (Nat. Hist.). Vl. Order of the Trustees, London, $532 \mathrm{pp}$.

- Handfield L, Handfield D (2010) Cucullia umbratica (Lepidoptera, Noctuidae), a new European noctuid in North America. ZooKeys 39: 183-186. https://doi.org/10.3897/ zookeys.39.426

- Heath J (Ed.) (1976) The moths and butterflies of Great Britain and Ireland. Volume I. Micropterigidae - Heliozelidae. The Curwen Press Ltd, London, 343 pp.

- Heath J, Emmet M (Eds) (1983) The moths and butterflies of Great Britain and Ireland. Volume 10. Noctuidae (Part II) and Agaristidae. Harley Books, Colchester, 459 pp.

- Hebert PDN, Penton EH, Burns JM, Janzen DH, Hallwachs W (2004) Ten species in one: DNA barcoding reveals cryptic species in the neotropical skipper butterfly Astraptes fulgerator. Proceedings of the National Academy of Sciences 101 (41): 14812-14817. https://doi.org/10.1073/pnas.0406166101

- Jinbo U (2017) List-MJ A checklist of Japanese moths. http://listmj.mothprog.com/. Accessed on: 2019-2-19.

- Jin Q, Hu X, Han H, Chen F, Cai W, Ruan Q, Liu B, Luo G, Wang H, Liu X, Ward R, Wu C, Wilson J, Zhang A (2018) A two-step DNA barcoding approach for delimiting moth species: moths of Dongling Mountain (Beijing, China) as a case study. Scientific Reports 8 (1). https://doi.org/10.1038/s41598-018-32123-9

- Kishida Y (Ed.) (2011) The standard of Moths in Japan 2. Gakken Education Publishing, Tokyo, 416 pp. [In Japanese].

- $\quad$ Kumar S, Stecher G, Tamura K (2016) MEGA7: Molecular Evolutionary Genetics Analysis Version 7.0 for Bigger Datasets. Molecular Biology and Evolution 33 (7): 1870-1874. https://doi.org/10.1093/molbev/msw054

- $\quad$ Price J (2017) The potential impacts of climate change on the biodiversity of Norfolk. Transactions of the Norfolk and Norwich Naturalists' Society 50 (1): 1-8.

- Scheffer SJ, Giblin-Davis RM, Taylor GS, Davies KA, Purcell M, Lewis ML, Goolsby J, Center TD (2004) Phylogenetic relationships, species limits, and host specificity of gallforming Fergusonina flies (Diptera: Fergusoninidae) feeding on Melaleuca (Myrtaceae). Annals of the Entomological Society of America 97 (6): 1216-1221. https:// doi.org/10.1603/0013-8746(2004)097[1216:prslah]2.0.c0;2 